\title{
ENTRY, STACKELBERG EQUILIBRIUM AND REASONABLE CONJECTURES*
}

\author{
Luis C. CORCHÓN and Félix MARCOS \\ Universidad Complutense de Madrid, 28023 Madrid, Spain
}

Final version received December 1987

We show that the consideration of Stackelberg equilibrium and reasonable conjecture equilibria (R.C.E.) provide some foundation to the concept of limit pricing.

\section{Introduction}

This paper deals with the problem of entry deterrence in two different ways. First, we analyze conditions under which entry prevention is the best strategy for an incumbent firm, and second, we link entry prevention with another important field of industrial organization, the analysis of reasonable conjectures.

That entry prevention may be of interest for incumbent firms has been studied by Osborne (1973), Spence (1977), Dixit (1979), Salop (1979), Milgrom and Roberts (1982) and Omori and Yarrow (1982). In all these papers equilibrium concept is some kind of Stackelberg equilibrium in which potential entrants behave in Cournot fashion and the incumbent firm is a Stackelberg leader. We follow this approach by widening the model to allow more general conjectures for entrants.

The theory of reasonable conjectures has been developed by Hahn (1978), in the framework of an exchange economy, and Hart (1982), in an oligopoly model with decreasing returns to scale. This theory attempts to endogenize conjectures assuming that firms choose those conjectures which maximize profits. 'Basically the conjectures of firms are reasonable if, given the conjectures of all other firms, no firm can increase its profit by departing from its own conjectures. That is, a resonable conjectural equilibrium is a Nash equilibrium where the strategies of firms are conjectures' [Hart (1982, p. 2)].

*The authors are grateful to two anonymous referees and to P.A. Geroski for helpful comments. Also, we would like to thank Ana Antón Pacheco for her linguistic advice. One of the authors is indebted to Oliver Hart for conversations about reasonable conjectures. The usual caveat applies. 
The idea that conjectures are the 'right' strategic space is quite appealing since conjectures can represent very different types of behaviour. However, Hart's results point out the existence of a large number of R.C.E. [Hart $(1985$, p. 129)]. In the final section we will argue that if economies of scale are considered, this may be changed.

Another interesting feature of R.C.E. is that, in such an equilibrium, conjectures must be locally correct [see Hart (1982) pp. 19-20]. This last requirement has been used in the literature under the name of consistent conjectural variations [see Bresnahan (1981), Perry (1982), Tanaka (1985) and Ulph (1983)]. Some of their results will be discussed in the final section.

In this paper we will prove that a kind of generalized Stackelberg equilibrium prevents the entry of any potential entrant (Proposition 1). Moreover under slightly stronger conditions, the limit price (see ahead definition 5) is a generalized Stackelberg equilibrium price (Proposition 2). Finally we analyze R.C.E. We do not obtain a complete characterization of the set of R.C.E. in our economy (which differs from Hart's paper (1982) in that economies of scale are present here). We will prove that the usual Stackelberg equilibrium is a R.C.E. That provides a rationale for the Sylos postulate (i.e., the assumption that potential entrants behave in a Cournot way). This is our Proposition 3. We end the paper with some comments on the significance of our results.

\section{Major definitions}

We will consider a homogeneous goods market in which there are $m$ firms. Firm 1 is the incumbent and firms $2,3, \ldots, m$ are potential entrants.

Let $x_{i}$ be the outpout of firm $i$ and $x=\sum_{i=1}^{m} x_{i}$. The price of the product is $p$. Let $p(x)$ be the inverse demand function which is assumed to be strictly decreasing. Profits for firm $i$ are $\pi_{i}\left(x_{i}, x_{-i}\right)=p(x) x_{i}-c_{i}\left(x_{i}\right), x_{-i}=x-x_{i}$ is the output of the remaining firms and $c_{i}\left(x_{i}\right)$ is the cost function of firm $i$ with $c_{i}(0)=0$. We will assume that $c_{i}\left(x_{i}\right)=c_{j}\left(x_{j}\right)$ if $x_{i}=x_{j} \forall i, j=2,3, \ldots, m$, i.e. they share the same cost function.

Definition 1. A conjecture for firm $i$ is a real valued function $\alpha_{i}\left(x_{i} ; \bar{p}, \bar{x}_{i}\right)$ defined $\forall x_{i}, \bar{x}_{i}, \bar{p} \in R+$.

The interpretation of a conjecture is that 'given a status quo position where the price is $\bar{p}$ and the firm is producing $\bar{x}_{i}$, firm $i$ conjectures that if it changed its supply to $x_{i}$, the equilibrium price would change to $\alpha_{i}\left(x_{i}, \bar{p}, \bar{x}_{i}\right)^{\prime}$ [Hart (1982)].

Definition 2. The correspondence $F_{i}\left(\bar{p}, \bar{x}_{i}\right)$ is said to be the reaction correspondence for firm $i=2,3, \ldots, m$ if 


$$
\begin{aligned}
F_{i}\left(\bar{p}, \bar{x}_{i}\right)= & \left\{\tilde{x}_{i} \in R_{+} \mid \alpha_{i}\left(\tilde{x}_{i}, \bar{p}, \bar{x}_{i}\right) \tilde{x}_{i}-c_{i}\left(\tilde{x}_{i}\right)\right. \\
& \left.\geqq \alpha_{i}\left(x_{i}^{\prime}, \bar{p}, \bar{x}_{i}\right) x_{i}^{\prime}-c_{i}\left(x_{i}^{\prime}\right) \forall x_{i}^{\prime} \in R_{+}\right\} .
\end{aligned}
$$

We will assume that firms $2,3, \ldots, m$ share the same conjecture. Therefore their reaction correspondences are identical.

Definition 3. A tuple $\left(x_{1}^{s}, \ldots, x_{m}^{s}, p^{s}\right)$ is said to be a Generalized Stackelberg Equilibrium (G.S.E.) with firm 1 as a leader if (a) $p^{s}=p\left(x_{1}^{s}+\cdots+x_{m}^{s}\right)$, (b) $\left(x_{i}^{s}, \ldots, x_{m}^{s}, p^{s}\right)$ maximizes $\pi_{1}\left(x_{1}, x_{-1}\right)$ subject to $x_{i} \in F_{i}\left(p^{s}, x_{i}^{s}\right), i=2,3, \ldots, m$.

That is, at a G.S.E. firms $2,3, \ldots, m$ maximize profits according to their conjectures and firm 1, subject to this constraint, maximizes its profits. Notice that if conjectures for firms $2,3, \ldots, m$ were of the Cournot type (see definition 7) a G.S.E. would be the usual notion of a Stackelberg equilibrium.

Definition 4. A price $\bar{p}$ is said to prevent entry if $\forall i=2,3, \ldots, m, 0 \in F_{i}(\bar{p}, 0)$.

Definition 5. A price $p^{1}$ is said to be the limit price if $p^{1} \geqq \bar{p} \forall \bar{p}$, both $\left(p^{1}\right.$ and $\bar{p}$ ) satisfying definition 4 . Notice that in this definition the value of $p^{1}$ depends on the conjecture of entrants.

Definition 6. A reasonable conjectural equilibrium is a tuple $\left(p^{*}, x_{1}^{*}, \ldots, x_{m}^{*}, \alpha_{1}^{*}, \ldots, \alpha_{m}^{*}\right)$ so that

(1) $p^{*}=p\left(x^{*}\right)$

(2) $x_{i}^{*}$ maximizes $\alpha_{i}^{*}\left(x_{i}, p^{*}, x_{i}^{*}\right) x_{i}-c_{i}\left(x_{i}\right) i=1,2,3, \ldots, m$

(3) $\forall i=1,2, \ldots, m$ there does not exist $\hat{p}, \hat{x}_{1}, \ldots, \hat{x}_{m}$, where $\hat{p}=p(\hat{x})$ and $\hat{x}_{j}$ fulfils (2) $\forall j \neq i$ so that $\pi_{i}(\hat{x})>\pi_{i}\left(x^{*}\right)$.

The previous definition is taken from [Hart (1982, pp. 5-6)]. Condition (2) requires $x^{*}$ to be a conjectural equilibrium, i.e., an equilibrium relative to conjectures $\alpha_{1}^{*}, \ldots, \alpha_{m}^{*}$. Condition (3) states that each firm is maximizing profits given the conjectures of the other firms.

The motivation for studying R.C.E. is 'The idea that firms do not care about the correctness of their conjectures per se, but only about whether they are maximizing profits. In a R.C.E. while there may be errors in a firm's conjecture, they are not of the sort that lead to reduce profits' [Hart (1982) pp.2)].

We end this section with a definition of a special kind of conjectures.

Definition 7. A conjecture is said to be of Cournot type if $\alpha_{i}\left(x_{i}, \bar{p}_{i}, \bar{x}_{i}\right)=$ $p\left[x_{i}-\bar{x}_{i}+p^{-1}(\bar{p})\right]$. 


\section{Results}

We add the following assumption that characterize structure of the industry:

Assumption I

(a) $c_{1}\left(x_{1}\right) / x_{1}$ is not increasing on $x_{1}$,

(b) $\exists i, x_{i}^{s}=0$,

(c) $x_{1}^{s}>0$.

Statement (a) is a way of introducing economies of scale; (b) implies that the number of potential entrants is large enough and (c) that the incumbent firm is active at the G.S.E.

Proposition 1. Given Assumption $1\left(x_{1}^{s}, \ldots, x_{m}^{s}\right)=\left(x_{1}^{s}, 0, \ldots, 0\right)$ i.e., $p^{s}=p\left(x_{1}^{s}\right)$ prevents entry

Proof. Suppose it is not so. Then $x_{1}^{s}, x_{2}^{s}, \ldots, x_{h}^{s}>0$ and $x_{h+1}^{s}, \ldots, x_{m}^{s}=0$ i.e., $0 \in F_{j}\left(p^{s}, 0\right), j=h+1, \ldots, m$.

Now, if firm 1 produces

$$
x_{1}=x_{1}^{s}+\cdots+x_{h}^{s}
$$

$p^{s}$ prevents entry by any other firm, i.e., $0 \in F_{i}\left(p^{s}, 0\right), i \neq 2, \ldots, m$. On the other hand profits for the incumbent

$$
x_{1}\left[p^{s}-\frac{c_{1}\left(x_{1}\right)}{x_{1}}\right]>x_{1}^{s}\left[p^{s}-\frac{c_{1}\left(x_{1}^{s}\right)}{x_{1}^{s}}\right] .
$$

are now bigger and, therefore $x_{1}^{s}$ does not fullfil (b) in Definition $3 .^{1}$

Now let us introduce an additional requirement in order to prove that limit price is a G.S.E. price. ${ }^{2}$

\section{Assumption 2}

(a) The profit function of the incumbent is quasiconcave in $x_{1}$

'Omori and Yafrow $(1982\}$ have proved a simitar proposition. They model a more general case for a market with a heterogeneous product, but their assumptions are somewhat stronger In particular we do nor require the incumbent firm to be identical to the potential entrants and we do not need fixed costs But the main difference is that in our case conjectures of the entrants are not necessarily Cournot, ie, the so called Sylos postulate is not necessary for our proof (see Section 4, 2).

${ }^{2}$ And hence $\left(x_{1}^{3}, 0, \ldots, 0\right)$, where $x_{1}^{1}=p^{-1}\left(p^{1}\right)$, is a G.S.E. 
(b) Limit price is lower than monopoly price.

Proposition 2. Under Assumptions 1 and 2, $p^{1}$ is a G.S.E. price.

Proof. Because of Definition 5 if the incumbent chooses $p^{1}$ then $0 \in F_{i}$ $\left(p^{1}, 0\right), i=2,3, \ldots, m$.

On the other hand, $p^{s}$ also prevents entry (Proposition 1), hence $p^{s} \leqq p^{1}$. If we call $x_{1}^{s}=p^{-1}\left(p^{s}\right)$ and $x_{1}^{1}=p^{-1}\left(p^{1}\right)$ then $x_{1}^{s} \geqq x_{1}^{1}$, and, from Assumption 2, $\pi_{1}\left(x_{1}^{s}, 0, \ldots, 0\right) \leqq \pi_{1}\left(x_{1}^{1}, 0, \ldots, 0\right)$, i.e., $x_{1}^{1}$ yields at least as much profit as $x_{1}^{1}$. This completes the proof. ${ }^{3}$

We conclude proving that the usual Stackelberg equilibrium notion is a R.C.E.

Proposition 3. Given Assumption 1, Stackelberg equilibrium is a R.C.E.

Proof. It is clear that $\left(x_{1}^{s}, 0, \ldots, 0\right)$ is an equilibrium relative to some conjectures, i.e., part 2) in Definition 6 is fulfilled. If firm 1 holds $x_{1}$ constant, then no firm $2,3, \ldots, m$ can make a profit since $x_{1}^{s}$ prevents entry. Therefore Cournot conjectures maximize profits for these firms (many other conjectures would possibly yield this conclusion as well). In addition, if firms $2,3, \ldots, m$ hold Cournot conjectures, firm 1 cannot do better than to choose the Stackelberg conjecture since, by its very definition, it maximizes profits for firm 1 if $2,3, \ldots, m$ hold their Cournot conjectures.

A similar proposition has been provided by D. Ulph (1983) for the case of $m=2$ (but Ulph uses a consistent conjectural equilibrium instead of a R.C.E.). However Ulph's proof does not generalize for $m>2$. This is so because the logic behind his proof is that once firm 1 has chosen $x_{1}^{s}$ (and the correspondent Stackelberg conjecture), the Cournot-type conjecture for firm 2 is locally consistent. However if a third firm with a Cournot-type conjecture is introduced, the above conjecture for firm 2 ceases to be consistent, since $x_{3}$ will, in general, change if $x_{2}$ is changed.

Finally note that Proposition 1 is robust to the introduction of more incumbents. Indeed, assuming that each incumbent behaves both as Cournot with respect to any other incumbent and as a generalized Stackelberg leader with respect to similar potential entrants, we can prove similar results to Proposition 1. In this sense, imperfect coordination among incumbents is irrelevant to show that G.S.E. prevents entry [for problems raised by such imperfect coordination, consult Gilbert and Vives (1984)]. However this equilibrium is not a R.C.E. since Cournot behaviour is seldom reasonable

${ }^{3}$ Obviously if $\pi_{1}(-)$ is strictly quasiconcave then $p^{s}=p^{1}$ is the unique G.S.E. price. 
when more than one firm is active (see the above discussion on Ulph's result when $m>2$ ).

\section{Final comments}

(I) Propositions 1 and 2 can be regarded as some kind of 'domino theorem'. Indeed the existence of an inactive potential competitor prevents entry for any potential entrants. The interpretation of this result is that when $m$ is small, limit pricing may be an expensive strategy (i.e., a low $p^{1}$ causes low profits). However if the number of potential competitors is large enough, the cost of limit pricing is small in comparison to the cost of having all these firms in the market. Therefore the right alternative for the incumbent firm is to prevent entry.

(2) The most common criticism of limit pricing theory is that it is based on an assumption (the Sylos postulate) that is not derived from the rational behaviour of agents. This paper refutes this view in two ways. First the so called Sylos postulate is not necessary for the theory. All we need is that potential entrants share the same conjecture and that at least one firm remain inactive. Second, the usual Stackelberg equilibrium is a R.C.E. and therefore rational in some sense.

(3) Even though our approach is static, Propositions 1 and 2 may be of interest to the dynamic theory of the dominant firm. Suppose we start stage zero with an incumbent and one potential entrant. Suppose also that the G.S.E. implies a positive output for the entrant [as may occur in Dixit (1979)]. Now suppose that a new competitor arrives at stage one and that G.S.E. again implies a positive output for this firm, and that in periods $2,3, \ldots$ we have identical results. Our Propositions 1 and 2 imply that at some stage the behaviour of the incumbent will change dramatically. Indeed, sooner or later, if the incumbent behaves as a Stackelberg leader, it will find incentives to fight (and to win) a price war against its competitors.

(4) Conjectures which are used in the proof of Proposition 3 can be shown to be consistent, i.e., potential entrants are right in their assumption that total output is fixed and the incumbent is right in assuming that potential entrants are of the Cournot type. Therefore our R.C.E. is in fact a consistent conjectural equilibrium of the kind discussed in the Introduction.

However our Proposition 3 is in conflict with the finding of some authors concerning the properties of consistent conjectural equilibrium (C.C.E.). For example it has been claimed that under increasing returns to scale no C.C.E. exists [see Bresnahan (1981) p. 939 and Perry (1982) p. 202]. But actually all they prove is that no symmetrical C.C.E. exists with more than one active firm. Our Proposition 3 implies that C.C.E. exists when only one firm in the market survives. Therefore putting together our Proposition 3 and the Bresnahan-Perry result we get the impression that under economies of scale 
C.C.E. and R.C.E. may imply strong restrictions for the equilibrium set: in fact the case may be that only one firm can be active.

Finally notice also that our analysis refutes the belief that in a situation of free entry only competitive equilibrium is a C.C.E. [see Perry (1982) and Tanaka (1985)].

(5) An interesting problem (which is not treated in our paper) is to study welfare associated with entry prevention and with competition. Some examples - obtained from the authors on request - show that the sum of consumer and producer surpluses in a linear economy, are larger under entry prevention that under competition à la Cournot in almost any possible case.

\section{References}

Bain, J.S., 1956, Barriers to new competition (Harvard University Press, Cambridge, MA).

Bresnahan, R.F., 1981, Duopoly models with consistent conjectures, American Economic Review $71,934-945$.

Dixit, A., 1979, A model of duopoly suggesting a theory of entry barriers, Bell Journal of Economics.

Gilbert, R. and X. Vives, 1984, Entry deterrence and the free rider problem, Mimeo.

Hahn, F., 1978, On non-Walrasian equilibria, Review of Economic Studies XLV.

Hart, O., 1982, Reasonable conjectures, Mimeo. (London School of Economics, London).

Hart, O., 1985, Imperfect competition in general equilibrium: An overview of recent work, in: Arrow and Honkapoha, eds., Frontiers of economics (Blackwell, Oxford).

Milgrom, P. and J. Roberts, 1982, Limit pricing and entry under incomplete information: An equilibrium analysis, Econometrica 50.

Omori, T. and G. Yarrow, 1982, Product diversification, entry prevention and limit pricing, Bell Journal of Economics, 242-248.

Osborne, D.K., 1973, On the rationality of limit pricing, Journal of Industrial Economics 22, no. 1 .

Perry, M., 1982, Oligopoly and consistent conjectural variations, Bell Journal of Economics, 197-205.

Salop, S.C., 1979, Strategic entry deterrence, American Economy Review 69.

Spence, M., 1977, Entry, capacity, investment and oligopolistic pricing, Bell Journal of Economics.

Sylos-Labini, P., 1962, Oligopoly and technical progress (Harvard University Press, Cambridge, MA).

Tanaka, Y., 1985, Consistent conjecture and free entry oligopoly: A general analysis, Economics Letters $17,15-18$.

Ulph, F., 1983, Rational conjectures in the theory of oligopoly, International Journal of Industrial Organization 1, 131-154. 